\title{
Purple membrane resists cell adhesion
}

\author{
ZHAO YingChun \& DING JianDong* \\ State Key Laboratory of Molecular Engineering of Polymers, Department of Macromolecular Science, Laboratory of Advanced Materials, Fudan \\ University, Shanghai 200433, China
}

Received August 4, 2011; accepted October 13, 2011

Bacteriorhodopsin is a well-known photoresponsive protein in the cell membrane of Halobacterium salinarum and exists in the patch called the purple membrane (PM). This letter reports, for the first time, its new function as a natural non-fouling substance for resisting cell adhesion. Mammalian cells such as murine preosteocytes MC3T3-E1 were seeded on the PM film. A significant resistance to cell adhesion on the film was found.

bacteriorhodopsin, purple membrane, cell adhesion, non-fouling, biomaterials

Non-fouling materials that resist cell adhesion are very important in fundamental research of cell-biomaterial interactions and some material applications and have thus been extensively investigated during the last decade [1-5]. Natural biomacromolecules, such as bovine serum albumin (BSA), have also conventionally been used to block cell adhesion [6,7]. This study reveals that the purple membrane (PM) of a natural photoresponsive protein, bacteriorhodopsin (BR), can serve as a new basic substance that resists adhesion of mammalian cells.

$\mathrm{BR}$ is a retinal-containing bacterial protein existing in the membrane patch called the PM [8], which is the simplest biological system for energy conversion. Excitation of BR with a photon causes a photocycle, resulting in uptake of a proton from the cytoplasmic side of the PM and release of another proton to the extracellular side. BR and relevant retinal proteins have attracted much attention as potential optical materials in 2-D or 3-D storage, holographic storage, optical filtering, light switching, neural networks, super-fast photo detection, motion detection and artificial retinas, etc [9-12] and also as model proteins in fundamental research [13-18].

To our knowledge, the PM has not been previously recognized as a non-fouling material for preventing cell adhe-

\footnotetext{
*Corresponding author (email: jdding1 @ fudan.edu.cn)
}

sion. Adhesion is the first cellular event that occurs when a cell comes into contact with a material surface, which influences subsequent cellular events such as proliferation and differentiation. Besides the studies of cell-material interactions for generating patterned surfaces of adhesion contrast, non-fouling surfaces are also useful for controlling cellular and bacterial adhesion in medical applications or under a bio-related complex environment. To date, a number of non-fouling substances have been reported, including poly (ethylene glycol) (PEG) or oligo(ethylene glycol) (OEG) [19-21] and PEG hydrogels [22-24], poly( $N$-isopropyl acrylamide) or its copolymers [25], and a globular protein, BSA [7]. This study affords a new choice of non-fouling substance, because, for the first time, the PM containing the membrane protein BR was found to resist cell adhesion.

PMs were purified from the Halobacterium salinarum strain $\mathrm{R}_{1} \mathrm{M}_{1}$ according to the standard procedure [26]. BSA was from Sinopharm Chemical Reagent Co., Ltd (Shanghai, China). Triethylene glycol momo-11-mercaptoundecyl ether, an OEG agent was from Sigma Aldrich (St. Louis, MO, USA). Conventional glass slides for biological labs obtained from Shanghai Jinglun Industry Glass Corporation (Shanghai, China) were used as the basic substrate. $\mathrm{H}_{2} \mathrm{SO}_{4}$ and $\mathrm{H}_{2} \mathrm{O}_{2}$ from Shanghai Heqi Co., Ltd (Shanghai, China) and ultrapure water produced in a Millipore apparatus (Academic A10 Milli-Q) were used to clean the glass slides. In 
preparation of BR-coated homogeneous surfaces, glass slides were first sprayed by gold. A PM suspension with a concentration of $10 \mathrm{mg} / \mathrm{mL}$ was then dropped onto the Au-coated glass and a film was formed after drying. The virgin $\mathrm{Au}$-coated surface promoted cell adhesion and served as a negative control for non-fouling surfaces. In the two positive control groups, a BSA aqueous solution, also with concentration of $10 \mathrm{mg} / \mathrm{mL}$, was dropped and dried on a piece of glass, resulting in a dense protein film, or thiolate-terminated OEGs were linked to gold by covalent bonds to form a self assembled monolayer.

Mammalian cells MC3T3-E1 were used to evaluate the fouling resistance effect of PM. Murine preosteoblasts MC3T3-E1 cells were seeded on the surfaces at a density $15000 \mathrm{cell} / \mathrm{cm}^{2}$. Cells were cultured in modified Eagle's medium- $\alpha$ (MEM- $\alpha$, Gibco) with $10 \%$ fetal bovine serum (FBS, Biochrome) in a humidified incubator at $37^{\circ} \mathrm{C}$ with a $5 \% \mathrm{CO}_{2}$ atmosphere. After 8-h cell culture, unattached cells were removed by PBS rinsing. The remaining cells were fluorescently stained by the Live/Dead Viability/Cytotoxicity Kit from Invitrogen Co., Ltd. Images of cells were taken in an inverted microscope (Zeiss Axiovert 200).

Compared with the Au-coated glass, the PM films significantly resisted cell adhesion of MC3T3-E1, as shown in Figure 1(a). Color images are shown in Figure S1. The Live/Dead straining led to green fluorescence of live cells and red fluorescence of dead cells. Most of cells were green in our experiments and it was rare to observe red cells. Thus, in addition to confirming the non-fouling property of PM, our cell experiments also indicate that PM is non-cytotoxic. The significant resistance of PM films to cell adhesion was further confirmed in the cases of murine fibroblasts NIH3T3 cells and rat mesenchymal stem cells (rMSC) (data not shown).

We also determined the statistics of density and spreading area of adherent MC3T3-E1 cells, with the results shown in Figure 1(b) and (c). All data were from analysis of low-magnification fluorescent micrographs via the ImageJ software package. Herein, the number of cells per unit area on the substrate indicates cell density, while cell spreading was quantified by the projected area of each cell on the substrate. A significant difference in cell density was found between the PM group and the other three groups. Cell spreading area exhibited a similar trend.

Further examination of the long-term resistance efficacy was carried out. The PM suspensions were dropped onto the Au-coated glass slide. PM films were formed in the center of a piece of Au-coated glass, and a tapered thin boundary of the BR film with center thickness $2 \mu \mathrm{m}$ was observed, as shown in Figure S2. Thus, an adhesion contrast was generated. Then, we seeded MC3T3-E1 cells on the heterogeneous surface. The cells gradually proliferated and fully covered the Au-coated area, as shown in Figure 2. Unattached cells were removed by PBS rinsing every day and the remaining cells were observed in an optical microscope. The PM film prevented intrusion of dense cells up to $14 \mathrm{~d}$, exhibiting a very persistent non-fouling capability. The purple color was retained in the center region, as shown in Figure S3. Thus, the PM films were stable under cell culture and were not easily washed out while exchanging the culture medium.

As is known, even OEG as the "gold standard" of a nonfouling substance maintains its resistance to cell adhesion after $7 \mathrm{~d}$ with difficulty, especially when the surrounding (a)
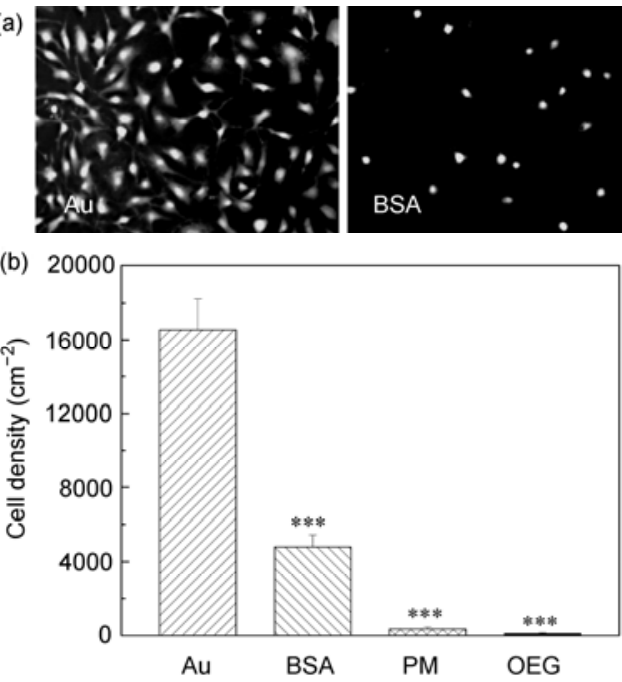
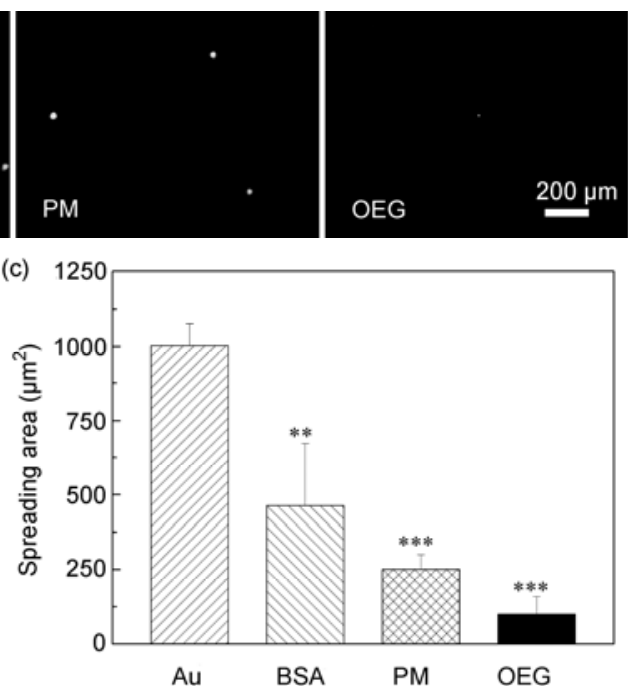

Figure 1 (a) Fluorescent micrographs of murine preosteocytes MC3T3-E1 on the glass surfaces modified with Au, BSA, PM, or OEG. The four surfaces in (a) are presented schematically in Scheme S1. The observations were made after 8-h culture. Statistical quantification of the density of attached cells and spreading area per attached cell is shown in (b) and (c). $n=3$; the $P$ values of Student $t$-tests for (b) and (c) are listed in Tables S1 and S2, respectively. The asterisks in (b) and (c) indicate significant differences compared with the negative control (the "Au" group): **, $P<0.01 ; * * *, P<0.005$. The corresponding colored images of (a) are shown in Figure S1. 

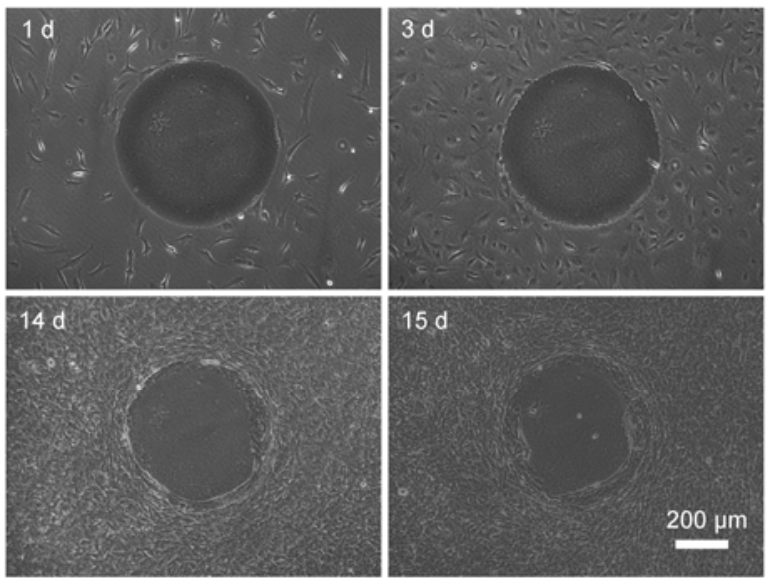

Figure 2 Optical micrographs of MC3T3-E1 cells on heterogeneous surfaces with a centered PM film on the Au-coated glass background. Cells were seeded on the whole surfaces. Images were captured after the indicated times. The corresponding colored images are shown in Figure S3.

cells have a high density [27]. In contrast to PEG and BSA etc, the PM films here remained photoresponsive (data not shown). It is well known that BR is very stable and is difficult to denature compared with most proteins $[9,10]$. Therefore, the finding of this study is non-trivial, considering both the strong anti-adhesion and photoresponse of PM.

PMs consist of lipids and BR at a molar ratio of about 10:1 and a weight ratio of about 25:75 [10], where lipids closely surround BR, as presented in Scheme S2. The protein trimers are arranged hexagonally in PM, and both cytoplasmic and extracellular sides of PM are negatively charged at neutral $\mathrm{pH}$ [28]. It has been known that lipids and negatively charged surfaces usually do not favor cell adhesion [29]. Therefore, the strong resistance by PM to cell adhesion may be mainly related to the negative surfaces and minor lipids. The actual mechanism remains an open question.

In summary, this letter reveals that the natural PM has a significant resistance to cell adhesion. The non-fouling property is very beneficial for potential applications of BR materials, for instance, as biosensors under complex environments. Our finding also affords an excellent model substance that resists cell adhesion, which could be useful in surface modification of biomaterials for regenerative medicine. The mechanism of prevention of non-specific protein absorption by PM is another open question. BR distinguishes itself from all of the currently known anti-fouling substances through its photoresponsive ability. It is worth noting that many retinal proteins, such as rhodopsin, halorhodopsin, xanthorhodopsin and archaerhodopsin [15,30], are light-driven ion pumps. Therefore, a direct extension of this work would be to examine possible non-fouling properties of the retinal proteins beyond BR. Resistance to other mammalian and non-mammalian cell types remain to be checked. Hence, the present report paves the way for further investigations and new applications of BR and possible other light-sensitive proteins.

This work was supported by the National Basic Research Program of China (2009CB930000, 2011CB606203) and the National Natural Science Foundation of China (21034002).

1 Ma H W, Hyun J H, Stiller P, et al. Adv Mater, 2004, 16: 338-341

2 Lavanant L, Pullin B, Hubbell J A, et al. Macromol Biosci, 2010, 10: 101-108

3 Yu Q A, Zhang Y X, Wang H W, et al. Acta Biomater, 2011, 7: $1550-1557$

4 Cao S, Wang J D, Chen H S, et al. Chin Sci Bull, 2011, 56: 598-612

5 Zhang Z, Ni J, Chen L, et al. Biomaterials, 2011, 32: 4725-4736

6 Koblinski J E, Wu M, Demeler B, et al. J Cell Sci, 2005, 118: 2965-2974

7 Lai Y X, Xie C, Zhang Z, et al. Biomaterials, 2010, 31: 4809-4817

8 Oesterhelt D, Stoeckenius W. Nat New Biol, 1971, 233: 149-152

9 Birge R R, Gillespie N B, Izaguirre E W, et al. J Phys Chem B, 1999, 103: $10746-10766$

10 Hampp N. Chem Rev, 2000, 100: 1755-1776

11 Xiang Y, Yang M, Su T, et al. J Phys Chem B, 2009, 113: 7762-7766

12 Zhao Y C, Ming M, Hong J, et al. Chin Sci Bull, 2010, 55: 3825-3830

13 Biesso A, Qian W, Huang X H, et al. J Am Chem Soc, 2009, 131: 2442-2443

14 Wu A G, Jia Z H, Schaper A, et al. Langmuir, 2006, 22: 5213-5216

15 Ming M, Lu M, Balashov S P, et al. Biophys J, 2006, 90: 3322-3332

16 Wu J, Ma D W, Wang Y Z, et al. J Phys Chem B, 2009, 113: 4482-4491

17 Chow B Y, Han X, Dobry A S, et al. Nature, 2010, 463: 98-102

18 Wang Y Z, Wu J, Ma D W, et al. Sci China Chem, 2011, 54: 405-409

19 Prime K L, Whitesides G M. Science, 1991, 252: 1164-1167

20 Huang J H, Grater S V, Corbellinl F, et al. Nano Lett, 2009, 9: 1111-1116

21 Chen Z L, Chen W, Yuan B, et al. Langmuir, 2010, 26: 17790-17794

22 Tang J, Peng R, Ding J D. Biomaterials, 2010, 31: 2470-2476

23 Yan C, Sun J G, Ding J D. Biomaterials, 2011, 32: 3931-3938

24 Sun J G, Tang J, Ding J D. Chin Sci Bull, 2009, 54: 3154-3159

25 Yu Q, Zhang Y X, Chen H, et al. Langmuir, 2010, 26: 8582-8588

26 Oesterhelt D, Stoeckenius W. Methods Enzymol, 1974, 31: 667-678

27 Luk Y Y, Kato M, Mrksich M. Langmuir, 2000, 16: 9604-9608

28 Alexiev U, Marti T, Heyn M P, et al. Biochemistry, 1994, 33: 298-306

29 Varki A. Nature, 2007, 446: 1023-1029

30 Balashov S P, Imasheva E S, Boichenko V A, et al. Science, 2005, 309: 2061-2064

Open Access This article is distributed under the terms of the Creative Commons Attribution License which permits any use, distribution, and reproduction in any medium, provided the original author(s) and source are credited. 